\title{
Constraining the spectral age of very asymmetric CSOs
}

\section{Evidence of the influence of the ambient medium}

\author{
M. Orienti ${ }^{1,2}$, D. Dallacasa ${ }^{1,2}$, and C. Stanghellini ${ }^{2}$ \\ 1 Dipartimento di Astronomia, Università di Bologna, via Ranzani 1, 40127 Bologna, Italy \\ e-mail: orienti@ira.inaf.it \\ 2 Istituto di Radioastronomia - INAF, via Gobetti 101, 40129 Bologna, Italy
}

Received 27 July 2006 / Accepted 25 September 2006

\begin{abstract}
Aims. We constrain the spectral ages for two very asymmetric Compact Symmetric Objects (CSO) from the B3-VLA-CSS sample, and we investigate the role of the ambient medium potentially able to influence the individual source evolution.

Methods. Multi-frequency VLBA observations have been carried out to study the distribution of the break frequency of the spectra across different regions of each source.

Results. From the analysis of synchrotron spectra and assuming an equipartition magnetic field, we find radiative ages of about $5 \times 10^{3}$ and $5 \times 10^{4}$ years for B0147+400 and B0840+424, respectively. The derived individual hot-spot advance speed is in the range between $0.03 c$ and $0.3 c$, in agreement with kinematic studies carried out on other CSOs. The very asymmetric morphology found in both sources is likely related to an inhomogeneous ambient medium in which the sources are growing, rather than to different intrinsic hot-spot pressures on the two sides.
\end{abstract}

Key words. galaxies: active - galaxies: evolution - radio continuum: galaxies - radiation mechanisms: non-thermal - galaxies: ISM

\section{Introduction}

Powerful and intrinsically compact $\left(\leq 1^{\prime \prime}\right)$ radio sources with convex radio spectra peaking between $100 \mathrm{MHz}$ and a few $\mathrm{GHz}$ represent a significant fraction $(\sim 15 \%)$ of the objects in flux density-limited radio source catalogues. When imaged with parsec-scale resolution, they often display a symmetric radio structure dominated by hot-spots and mini-lobes, namely a scaled-down version (from 0.1 to a few $\mathrm{kpc}$ ) of powerful, edgebrightened radio galaxies known as classical doubles. These radio sources, termed "Compact Symmetric Objects" (CSOs) by Wilkinson et al. (1994), are currently considered the early stages of the large radio sources (Fanti et al. 1995; Readhead et al. 1996; Snellen et al. 2000).

Two independent pieces of evidence strongly support the youth scenario: the kinematic study of hot-spot separation velocities (e.g. Polatidis \& Conway 2003; Gugliucci et al. 2005), and the measure of radiative age from the analysis of the source spectrum (Murgia 2003; Murgia et al. 1999); both find ages of about $10^{3}-10^{5}$ years.

On the other hand, the alternative model, the frustration scenario (Baum et al. 1990; van Breugel et al. 1984), which postulates that the radio source is trapped by an unusually dense gas, lacks any observational evidence, at least for the CSOs observed so far (see e.g. Fanti et al. 2000; Siemiginowska et al. 2005).

Synchrotron spectral ageing is based on the determination of the spectral break, which occurs at progressively lower frequencies as time passes. Indeed, according to various source growth models (i.e. Kardashev 1962; Pacholczyk 1970; Jaffe \& Perola 1974), relativistic electrons located in different source regions have been deposited at different times, and locally the electron age measures the time elapsed since their production and/or latest acceleration $\left(t_{\text {syn }}\right)$ when they crossed the hot-spot during its outward motion. The radiative age can be easily computed once the break frequency $\left(v_{\mathrm{br}}\right)$ and the magnetic field $(B)$ are known:

$t_{\mathrm{syn}} \propto B^{-3 / 2} v_{\mathrm{br}}^{-1 / 2}$

So far, most if not all the works on the measure of the source radiative age are based on the spectral break derived from the source-integrated spectra. One disadvantage of this approach is that the contributions of the various source components (core, jets, hot-spots and lobes), each one with its own spectral shape, are all mixed together. The brightest component is the one that influences the age determination the most; if we consider a source whose emission is dominated by the hot-spots where electrons are likely to be re-accelerated, the spectral ages derived can be completely unrelated to the source age.

On the other hand, the radiative age of the back-flow tail in the lobes measures the time elapsed since the last acceleration of those particles, namely when the hot-spot crossed that location during its outward expansion. The best (i.e. oldest) measure of the radiative age then comes from the innermost edges of the lobes, where the electrons were deposited at the very beginning.

Multi-frequency NRAO VLBA images can be very effective in constraining the source age in small radio sources whose radio emission contains a substantial contribution from the mini-lobes. Using multi-frequency images with pc-scale resolution it is possible to study the spectral ageing in intrinsically very small radio galaxies: it is important to distinguish regions in which electrons are injected/accelerated (i.e. core and hot-spots) from those in which electrons age, like in the back-flow tails of the mini radio lobes.

This paper reports the results of new multi-frequency $L$ (1.4$1.6 \mathrm{GHz}), C(4.5-4.9 \mathrm{GHz})$ and $X(8.1-8.5 \mathrm{GHz})$ bands) VLBA observations of two CSOs from the B3-VLA-CSS sample 
Table 1. Basic information on the VLBA observations: Col. 1: source name (B1950); Col. 2: Observed frequency; Col. 3: Date of observations; Col. 4: missing antenna; Cols. 5 and 6: shortest and longest projected baselines; Cols. 7-9: beam major and minor axis and PA of the full resolution images; Col. 10: $1 \sigma \mathrm{rms}$ noise level on the full resolution image plane.

\begin{tabular}{cccccccccc}
\hline \hline $\begin{array}{c}\text { Source } \\
\text { B1950 } \\
(1)\end{array}$ & $\begin{array}{c}v H z \\
\text { GHz }\end{array}$ & Obs. date & $\begin{array}{c}\text { Missing } \\
\text { antennas } \\
(4)\end{array}$ & $\begin{array}{c}u_{\min } \\
\mathrm{M} \lambda \\
(5)\end{array}$ & $\begin{array}{c}u_{\max } \\
\mathrm{M} \lambda \\
(6)\end{array}$ & $\begin{array}{c}\text { mas } \\
(7)\end{array}$ & $\begin{array}{c}\text { Beam } \\
\text { mas } \\
(8)\end{array}$ & $\begin{array}{c}\text { o } \\
(9)\end{array}$ & $\begin{array}{c}\text { Noise } \\
\text { my/beam } \\
(10)\end{array}$ \\
\hline B0147+400 & 1.407 & $09 / 06 / 2004$ & & 0.16 & 41 & 9.3 & 5.3 & -3 & 0.21 \\
& 1.643 & & & 0.18 & 50 & 8.3 & 4.7 & -4 & 0.38 \\
& 4.543 & & & 0.49 & 110 & 2.9 & 1.7 & -7 & 0.11 \\
& 4.893 & & & 0.50 & 140 & 2.7 & 1.6 & -5 & 0.10 \\
& 8.115 & & & 0.99 & 230 & 1.7 & 1.0 & 5 & 0.08 \\
B0840+424 & 8.493 & & & 1.05 & 250 & 1.6 & 0.9 & 5 & 0.07 \\
& 1.407 & $11 / 24 / 2004$ & $\mathrm{HN}$ & 0.15 & 40 & 12.0 & 7.5 & -2 & 0.19 \\
& 1.643 & & $\mathrm{HN}$ & 0.18 & 46 & 10.5 & 6.9 & 0 & 0.17 \\
& 4.543 & & $\mathrm{SC}$ & 0.48 & 110 & 3.5 & 1.9 & -20 & 0.15 \\
& 4.893 & & $\mathrm{SC}$ & 0.50 & 125 & 3.4 & 1.8 & -20 & 0.14 \\
& 8.115 & & $\mathrm{SC}$ & 0.83 & 210 & 2.0 & 1.6 & -23 & 0.16 \\
& 8.493 & & $\mathrm{SC}$ & 0.88 & 230 & 2.0 & 1.5 & -18 & 0.12 \\
\hline
\end{tabular}

(Fanti et al. 2001): B0147+400 and B0840+424. For both sources the core has been clearly detected.

Throughout this paper, we assume $H_{0}=71 \mathrm{~km} \mathrm{~s}^{-1} \mathrm{Mpc}^{-1}$, $\Omega_{\mathrm{M}}=0.27$ and $\Omega_{\lambda}=0.73$, in a flat Universe.

\section{Observations and data reduction}

Pc-scale resolution observations were carried out in two different runs using the VLBA plus a single VLA antenna, on September 6th $(\mathrm{B} 0147+400)$ and November 24th 2004 $(\mathrm{B} 0840+424)$, in full polarization mode with a recording bandwidth of $16 \mathrm{MHz}$ at $128 \mathrm{Mbps}$, for a total of $18 \mathrm{~h}$. Each source was observed for about 100, 150 and $210 \mathrm{~min}$ in $L, C$ and $X$ bands respectively, spread into short scans at various hour angles to improve the $u v$ coverage. In order to achieve a wider frequency coverage, in each observing band we observed with two $8-\mathrm{MHz}$ sub-bands widely spaced in frequency, obtaining 6 independent images, which improve the ability to constrain the spectral models. Details on the observations are summarized in Table 1.

The correlation was performed at the VLBA correlator in Socorro, and data reduction was carried out with the NRAO AIPS package. After the application of system temperature and antenna gain information, the amplitudes were checked using the data on DA $193(\mathrm{~J} 0555+398)$ which is unresolved on a large subset of baselines at all frequencies, and whose flux density is monitored at the VLA in $C$ and $X$ bands.

In both observations the sources DA 193 and J0927+3902 were both used to generate the bandpass correction.

The error on the absolute flux density scale can be estimated within $3-5 \%$ on the basis of the fluctuations of the amplitude gain solutions.

In $C$ and $X$ bands the instrumental polarization was removed by using the AIPS task PCAL; the absolute orientation of the electric vector of DA 193 and J0927+3902 was compared with the VLA/VLBA polarization calibration database to derive the corrections. The values derived from the two sources were in excellent agreement $\left(\leq 2^{\circ}\right)$. The calibration of the instrumental polarization was not performed for the $L$ band data.

The images at each individual frequency were obtained after a number of phase-only self-calibration iterations. Information on the full resolution Stokes $I$ images is given in Table 1 . Stokes $U$ and $Q$ images were produced from the final dataset.
As last step, we produced images at 1.6, 4.5, 4.9, 8.1 and $8.5 \mathrm{GHz}$, using natural grid weighting and the same $u v$-range common to all the observing frequencies for each source (1.05-41 M $\lambda$ and $0.88-40 \mathrm{M} \lambda$ for $\mathrm{B} 0147+400$ and B0840+424 respectively), in order to have almost the same $u v$ and image sampling, as well as restoring beam, as for the $1.4 \mathrm{GHz}$ data.

For each source, these low-resolution images were combined to produce a multi-frequency data cube, which was then analyzed by the synage++ software (Murgia 2000) for subsequent spectral studies. Image registration was checked by comparing the location of optically thin bright features. In this paper, we do not show the full-resolution images since they do not add any new information to those presented by Dallacasa et al. (2002) and Orienti et al. (2004).

\section{Multi-frequency spectral analysis}

Spectral index imaging is quite a hard task for VLBI experiments, since it is difficult to obtain well matched $u v$-coverages at the various observing frequencies. In particular there is a lack of short spacings at high frequencies.

In our VLBA observations, the key addition of a single VLA antenna makes the differences in the sampling density at short spacing less effective, allowing us to produce high-resolution spectral index images. Furthermore, the availability of 6 independent frequencies allows us to determine the source age by fitting the spectra in each pixel, with a good confidence level.

The multi-frequency high-resolution images provided by VLBA allow the determination of the nature of the source components (core, jets, lobes and hot-spots) and therefore, allow us to choose the best model to fit the observed spectral shapes. Indeed, although the radiative losses always imply a high-frequency steepening, the local spectral shape is strongly related to the evolution of the emission from an electron population and by the possible presence of injection of fresh relativistic electrons. For example, in the hot-spots, we expect that the observed spectra are well fitted by models predicting a continuous injection of fresh particles, while lobes and extended features will be better fitted by single-injection models (i.e. JP, Jaffe \& Perola 1974; KP, Kardashev 1962; Pacholczyk 1970) where the radiative losses play an important role in modifying the initial spectral shape. 
A previous work on the spectral ageing in two CSOs (Murgia 2003) has shown that the break frequency decreases if we move from regions near the hot-spot toward those located at the inner edges of the lobe, in the core direction. This is consistent with the dynamical scenario in which the electrons deposited at the centre of the source are older than those found closer to the hotspot, which is what is expected if the source is expanding with time and the principal site of particle acceleration is the hot-spot.

Therefore, to obtain a measure of the source age, it is possible to investigate how the break frequency changes across the lobes, where electrons age without any further substantial acceleration and, locally, without any new supply of fresh particles.

\section{Multi-frequency images of CSOs: constraining the age from the spectra}

As mentioned in Sect. 3, the pc-scale resolution achieved by the VLBA enables us to study extremely compact source components, found to be unresolved with other radio telescopes. We perform a detailed spectral ageing study of the CSO sources B0147+400 and B0840+424 from the B3-VLA-CSS sample (Fanti et al. 2001). Both radio sources are characterized by a weak core and two very asymmetric, both in arm-length ratio and brightness, well-resolved mini-lobes. In B0147+400 the two mini-lobes lie roughly in the East-West direction, and are connected by an extended, steep-spectrum $(\alpha>2)$ bridge visible in the $L$ band only, while in B0840+424 the mini-lobes are deployed roughly in the North-South direction.

In the standard source model, the brightest component is also the farthest from the core, since the differences in brightness and arm-length ratio are due to beaming effects and path delay. In radio galaxies where the radio axis is oriented at large angles to the line of sight, such asymmetries are expected to be quite small. On the contrary, in the two sources in this paper, the brightest lobe is the one closest to the core, while the faintest one is much further away, suggesting a strong influence exerted by the ambient medium, which can be quite complex and inhomogeneous on such small scales. For a more detailed description of the morphologies and the physical parameters of both sources, see Orienti et al. (2004) and Dallacasa et al. (2002).

\subsection{The hot-spots}

The radio source $\mathrm{B} 0147+400$ displays two rather compact features that can be interpreted as hot-spots (labeled C in Fig. 1), located $\sim 9$ mas South-East and $\sim 55$ mas North-West of the core, with a flux density ratio of $S_{\mathrm{SE}} / S_{\mathrm{NW}} \sim 2.1$ and 5.2 at $C$ and $X$ bands (Orienti et al. 2004).

The brightest hot-spot (labeled SH in Fig. 1) is embedded in the SE component and, with its flux density (208, 158 and $121 \mathrm{mJy}$ at $L, C$ and $X$ band respectively, Dallacasa et al. 2002; Orienti et al. 2004), dominates the radio emission ( $\sim 68 \%)$ in $C$ and $X$ bands. It is best fitted by a power-law with an injection spectral index of $\alpha_{\text {inj }} \sim 0.40$. This implies that there is a continuous supply and re-acceleration of fresh relativistic electrons, in agreement with what is predicted by the source growth models.

On the other hand, the faint hot-spot embedded in the $\mathrm{NW}$ component (labeled NH) is well fitted by a higher injection spectral index of $\alpha_{\text {inj }} \sim 0.65$.

In the source B0840+424 the Northern and Southern components are $\sim 15$ and $\sim 100$ mas apart from the core (labeled $\mathrm{C}$ in Fig. 1), with a flux density ratio of $S_{\mathrm{N}} / S_{\mathrm{S}} \sim 6.0$ and 7.1 at $C$ and $X$ bands (Orienti et al. 2004).
The brightest hot-spot (labeled NH) is located at the centre of the Northern component. The hot-spot can only be fitted by a single-injection model, with an $\alpha_{\text {inj }}$ of $\sim 0.40$, similarly to what is found in the bright hot-spot of B0147+400. However, this can be easily explained by considering a strong contamination by the lobe emission. The hot-spot embedded in the Southern component (labeled SH) is not bright and well-defined as in the Northern lobe, and it displays a higher injection spectral index of $\alpha_{\text {inj }} \sim 0.50$.

In both sources, the faintest hot-spots have $\alpha_{\text {inj }}$ steeper than what is found by Orienti et al. (2004). However, that work, based on 3 independent frequencies only, provided good fits for a range of injection spectral indices. The availability of 6 independent spectral points in this study allows us to set stronger constraints on the fits and their parameters.

\subsection{The lobes}

The lobes represent the ideal loci where the radiative age can be computed with a high degree of accuracy. To estimate the source age, we determine the variation of the break frequency across different regions of the extended components, such as the lobes. To improve the reliability of the analysis, we consider only those regions with a good signal-to-noise ratio at all frequencies. Indeed, at high frequencies (i.e. $X$ band), low surface brightness regions are almost completely resolved out, causing the fits to fail.

Since the electrons in the lobes have likely received the last acceleration in the corresponding hot-spot, we fit their spectra with a single-injection model with the $\alpha_{\text {inj }}$ derived for the hot-spot.

We try to fit the spectra with both the JP and KP models. The JP model assumes that the pitch angle $\theta_{\mathrm{p}}$ between the electron velocity and the magnetic field direction is continuously reisotropized, making the electrons age in the same way. In the $\mathrm{KP}$ model, the pitch angles of the electrons populations are constant, making electron populations with different $\theta_{\mathrm{p}}$ age in different ways.

Although both models provide similar fits to our spectra, the fits with the JP model have smaller reduced $\chi^{2}$ than the KP.

In B0147+400, we studied the break frequency across the Northern lobe (labeled NL). Using the injection spectral index of $\alpha_{\mathrm{inj}}=0.65$ as derived for the northern hot-spot, we find a minimum break frequency $v_{\mathrm{br}}=14 \mathrm{GHz}$.

In the case of B0840+424, we fitted the spectra across the Southern lobe (labeled SL) with a JP model with $\alpha_{\text {inj }}$ of 0.50 , obtaining the lowest break frequency of $7.4 \mathrm{GHz}$.

For both sources, we have considered different regions across the lobe, and the final choice was made on the best compromise between the largest distance from the hot-spot and the need for significant emission at the highest frequencies.

\subsection{The cores}

In both $\mathrm{B} 0147+400$ and $\mathrm{B} 0840+400$ the core, labeled $\mathrm{C}$ in Fig. 1, has been definitely identified by means of the inverted spectrum displayed $(\alpha \sim-0.5$ and -0.03 for B0147+400 and B0840+424, respectively; Orienti et al. 2004).

These compact components are better visible in the full resolution images at the highest observing frequency. Indeed, in the $X$ band they account for almost $10 \%$ of the total flux density of the whole source. 



Fig. 1. The spectral fits in the lobes and hot-spots of B0147+400 and B0840+424. On each fit we report the model, the injection spectral index, the break frequency and reduced-chi-squared. The upper panel shows, clockwise, the local spectra in the Northern lobe (NL), Northern hot-spot $(\mathrm{NH})$ and Southern hot-spot $(\mathrm{SH})$ of the source B0147+400. The bottom panel shows, clockwise, the local spectra in the Norther hot-spot (NH), Southern hot-spot (SH) and Southern lobe (SL) of the source B0840+424. The spectra of the core components are not shown, since they can be found in Orienti et al. (2004). The restoring beam $(H P B W)$ is $8.20 \times 4.46$ mas in PA -3.59 and $1.198 \times 0.753$ in PA -1.94 for B0147+400 and B0848+424 respectively.

Neither core shows any significant flux density variability in the $X$ band in our data.

\subsection{Radiative ages and the nature of CSOs sources}

From Eq. (1), it is clear that the synchrotron age is strictly related to the break frequency $v_{\mathrm{br}}$, which can be derived from the fits to 
Table 2. Source ages. Column 1: the source name; Col. 2: the redshift; Col. 3: the total linear size; Col. 4: the equipartition magnetic field, computed assuming the source parameters from Orienti et al. (2004); Col. 5: the radiative age, computed using Eq. (1) and the lowest break frequency as described in Sect. 4.2; Col. 6: the projected linear size, in pc, between the region considered to derive radiative age and the location of the corresponding hot-spot; Col. 7: the projected distance, in pc, between the core component and the faintest hot-spot; Col. 8: the mean advance speed of the faintest hot-spot; Col. 9: the source age; Col. 10: the projected distance between the core and the brightest hot-spot; Col. 11: the mean advance speed of the brightest hot-spot.

\begin{tabular}{|c|c|c|c|c|c|c|c|c|c|c|}
\hline Source & (2) & $\begin{array}{c}\text { LLS } \\
\text { pc } \\
(3)\end{array}$ & $\begin{array}{c}B_{\text {eq }} \\
m G \\
(4)\end{array}$ & $\begin{array}{c}t_{\mathrm{syn}} \\
10^{3} \mathrm{yr} \\
(5)\end{array}$ & $\begin{array}{l}\mathrm{LS} \\
\mathrm{pc} \\
(6)\end{array}$ & $\begin{array}{c}\text { Dist }_{\text {fhs }} \\
\text { pc } \\
(7)\end{array}$ & $\begin{array}{c}v_{\text {fhs }} \\
c \\
(8) \\
\end{array}$ & $\begin{array}{c}\text { Age } \\
10^{3} \mathrm{yr} \\
(9)\end{array}$ & $\begin{array}{c}\text { Dist }_{\text {bhs }} \\
\text { pc } \\
(10)\end{array}$ & $\begin{array}{c}v_{\mathrm{bhs}} \\
\mathrm{c} \\
(11)\end{array}$ \\
\hline \multirow[t]{6}{*}{ B0147+400 } & 0.20 & 210 & 3.0 & 2.6 & 64 & 188 & 0.08 & 7.5 & 29 & 0.01 \\
\hline & 0.35 & 316 & 3.0 & 2.6 & 97 & 258 & 0.13 & 7.5 & 44 & 0.02 \\
\hline & 0.40 & 344 & 3.0 & 2.6 & 106 & 307 & 0.13 & 7.5 & 48 & 0.02 \\
\hline & 0.60 & 429 & 3.3 & 2.2 & 132 & 383 & 0.19 & 6.5 & 60 & 0.03 \\
\hline & 0.80 & 488 & 3.6 & 2.0 & 149 & 432 & 0.25 & 5.6 & 67 & 0.04 \\
\hline & 1.00 & 518 & 4.0 & 1.7 & 159 & 462 & 0.31 & 4.8 & 72 & 0.05 \\
\hline \multirow[t]{6}{*}{ В0840+424 } & 0.20 & 362 & 1.2 & 14.0 & 64 & 301 & 0.015 & 70.0 & 49 & 0.002 \\
\hline & 0.35 & 544 & 1.6 & 9.4 & 97 & 452 & 0.034 & 44.0 & 73 & 0.005 \\
\hline & 0.40 & 592 & 1.6 & 9.1 & 106 & 492 & 0.038 & 42.0 & 79 & 0.006 \\
\hline & 0.60 & 740 & 1.7 & 8.3 & 132 & 614 & 0.052 & 38.0 & 100 & 0.009 \\
\hline & 0.80 & 834 & 1.9 & 7.0 & 149 & 692 & 0.069 & 32.5 & 112 & 0.011 \\
\hline & 1.00 & 892 & 2.1 & 6.0 & 159 & 740 & 0.086 & 28.0 & 120 & 0.014 \\
\hline
\end{tabular}

the observed radio spectrum, and the magnetic field. Direct measurements of the magnetic field are very difficult, often impossible to carry out. Ideally, it can be measured from the turnover frequency and component sizes, if both are known, but the uncertainties remain quite large. Alternatively, we can measure it by comparing synchrotron and inverse Compton losses, but X-ray observations of small and young radio sources have not provided strong constraints so far.

In this paper, the magnetic field of the source components has been computed assuming minimum energy and equipartition conditions, and using standard formulae (Pacholczyk 1970). Furthermore, proton and electron energies have been assumed to be equal, with a filling factor of unity (i.e. the source volume is fully and homogeneously filled by relativistic plasma); an ellipsoidal geometry and an average optically thin spectral index of 0.7 have been adopted. The observational parameters involved, such as the flux density and the projected linear size of the source components, are from Orienti et al. (2004).

We also point out that the magnetic field of CSS sources is of a few orders of magnitude higher than that "equivalent" to the Cosmic Microwave Background radiation photons. Inverse Compton losses are, therefore, neglected, the synchrotron being the main cooling mechanism.

Furthermore, even adiabatic losses are negligible, since the energy spent due to the adiabatic expansion is three orders of magnitude smaller than the synchrotron emission.

Unfortunately, both sources lack spectroscopic redshifts. The source B0147+400 has no optical identification, while B0840+400 can be identified with a galaxy in the Sloan Digital Sky Survey (SDSS). With the magnitudes provided by the SDSS for the source B0840+424, we made use of the HyperZ code (Bolzonella et al. 2000) to infer the photometric redshift. We obtained a photometric redshift of 0.35 , with a probability of $83 \%$.

Since most of the intrinsic physical parameters, such as the magnetic field $\left(B_{\text {eq }}\right)$ and the linear size (LS), critically depend on the redshift, for each source we provide a set of values considering a few cases in which $z$ is in the range of $0.2-1.0$ (Table 2). The radiative ages found in this way are in the range of $10^{3}-10^{4}$ years. However, as previously mentioned, this should not be considered as the source age. Models of source evolution predict that relativistic electrons are deposited in the region of last acceleration, where they age, while the hot-spot continues through the interstellar medium. Therefore, the electrons considered for the previous computation have no memory of the original injection by the core, since they have already been reaccelerated by the hot-spot. The radiative ages derived give us an indication of the time elapsed since the last acceleration in the hot-spot.

Measuring the distance between the region considered for the radiative age and the hot-spot, we can estimate the hot-spot advance speed. In both sources, the hot-spot has covered a distance of $\sim 20$ mas from the region where we could measure the radiative age, which implies a range of mean hot-spot velocities of $0.08 c$ to $0.31 c$ for B0147+400 and between $0.038 c$ and $0.086 c$ for $\mathrm{B} 0840+424$, depending on the source redshift (Table 2). Since we know the distance between the hot-spot and the core component, and assuming a mean hot-spot velocity over the whole lifetime of the source (Polatidis \& Conway 2003), we can constrain the true source age. In the case of B0147+400, the core-hot-spot separation is 53 mas, which leads to a source age in the range of $4.8 \times 10^{3}$ and $7.5 \times 10^{3}$. For the source B0840+424, the distance between the core and the hot-spot is larger ( 97 mas), and we derive ages between $2.8 \times 10^{4}$ and $7.0 \times 10^{4}$ years, $\left(4.4 \times 10^{4}\right.$ years, considering the photometric redshift of 0.35$)$.

We can also estimate the mean advance speed of the brightest hot-spot, dividing its distance from the core by the source age. We obtain velocities ranging from $0.01 c$ to $0.05 c$ and $0.002 c$ and $0.014 c$ for the brightest hot-spots of B0147+400 and B0840+424 respectively (Table 2). With such velocities, it is not possible to detect any hot-spot advance over a short period of time. We compared the data at the two epochs available, observed about three years apart, by means of the MODELFIT and the JMFIT, but we did not find any significant variation in the position of the most compact source components. The accuracy in the determination of the component position is in agreement with the above results.

\subsection{Linearly polarized emission}

Images in the $U$ and $Q$ Stokes' parameters have been derived for both the target sources, as well as for the calibration objects in $C$ and $X$ bands. Calibration sources proved to have 
integrated VLBA polarized emission in agreement with VLA measurement carried out at a very near epoch, as available from the VLA/VLBA polarization calibration database.

No significant ( $\geq 3 \sigma$ noise level) polarized emission was detected for the target sources in $C$ and $X$ bands, consistent with previous VLA observations (Fanti et al. 2001) at the same frequencies, where both sources appear unresolved and unpolarized at a resolution of $\sim 0$. $^{\prime} 4$.

Polarization images are not shown.

The local upper limits we could infer are of $0.03 \%$ and $0.14 \%$ for the Southern and Northern components of $\mathrm{B} 0147+400$ respectively, and of $0.01 \%$ and $0.08 \%$ for the Northern and Southern components of B0840+424.

Our results are in good agreement with those found by Fanti et al. (2004). In fact, although we do not know the redshift of both sources, we can estimate their projected linear sizes $\leq 520$ and $900 \mathrm{pc}$ for B0147+400 and B0840+424, respectively (Table 2). Following the work of Fanti et al. (2004), both sources fall in the interval of unpolarized CSS sources even in the $X$ band, suggesting that the source size is not large enough to lead the radio emission to emerge out of the "Faraday screen".

\section{Discussion}

From the analysis of the synchrotron spectra in two CSOs, we estimate radiative ages of about $5 \times 10^{3}$ and $5 \times 10^{4}$ years, in good agreement with kinematic and radiative studies carried out on the same class of objects (Polatidis \& Conway 2003; Murgia et al. 1999). Our measurements are not aimed to find the "accurate" source age (which may be revised as new measurements are added), but rather to determine whether the target sources are to be considered young radio galaxies.

The approach used to infer the source age, described in Sect. 4.3, is based on the strong assumption that the hot-spot velocity derived is truly representative of the mean individual hot-spot advance speed. However, there are several mechanisms that would cause the instantaneous hot-spot speed to vary, such as hydrodynamically introduced internal pressure changes (Norman 1996), as well as an inhomogeneous external medium.

The large asymmetries both in arm-ratio and brightness shown by $\mathrm{B} 0147+400$ and $\mathrm{B} 0840+424$ strongly suggest that at least one of the two aforementioned possibilities applies. In Table 3, we report the hot-spot internal pressures computed assuming that the source components are in the minimum energy condition. Contrary to expectations, we find that in both sources the hot-spot with the highest pressure is the slowest one, suggesting that the asymmetric morphology is more likely due to an inhomogeneous clumpy ambient medium.

Using simple one-dimensional ram-pressure arguments, the advance speed $v$ of the hot-spot is determined by the equilibrium between the internal pressure $p_{\mathrm{i}}$ and the ram-pressure of the external medium:

$p_{\mathrm{i}} \propto n_{\mathrm{ext}} m_{\mathrm{p}} v^{2}$

where $n_{\text {ext }}$ is the particle density of the external medium and $m_{\mathrm{p}}$ is the proton mass. We assume an external density profile of the King type, as suggested by X-ray observations of early-type galaxies (i.e. Trinchieri et al. 1986). Since the total linear sizes of both sources are smaller than the core radius $(<1 \mathrm{kpc})$, we can assume a roughly constant external gas density.

If in Eq. (2) we consider the average hot-spot velocity (Table 2) and the minimum pressure (Table 3), computed at an indicative redshift of 0.35 , for both sources we find that the
Table 3. The hot-spot pressure. Column 1: the source name; Col. 2: the source component; Col. 4: VLBA flux density at $8.4 \mathrm{GHz}$; Cols. 5, 6: deconvolved angular size of the major and minor axis of the source component; Col. 7: the minimum pressure, computed assuming the minimum energy condition, and an indicative redshift of 0.35 .

\begin{tabular}{cccccc}
\hline \hline Source & Component & $\begin{array}{c}S_{8.4} \\
\text { mJy }\end{array}$ & $\begin{array}{c}\theta_{\text {maj }} \\
\text { mas }\end{array}$ & $\begin{array}{c}\theta_{\min } \\
\text { mas }\end{array}$ & $\begin{array}{c}p_{\min } \\
\text { dyne } / \mathrm{cm}^{2}\end{array}$ \\
\hline B0147+400 & HS & 119 & 3 & 2 & $3.4 \times 10^{-6}$ \\
& HN & 22 & 9 & 6 & $4.9 \times 10^{-7}$ \\
B0840+424 & HN & 249 & 8 & 3 & $2.1 \times 10^{-6}$ \\
& HS & 26 & 13 & 8 & $1.4 \times 10^{-7}$ \\
\hline
\end{tabular}

brightest and closest hot-spot is likely digging its way through a quite dense medium $\left(n_{\mathrm{ext}} \sim 5.7\right.$ and $50.0 \mathrm{~cm}^{-3}$, for B0147+400 and $\mathrm{B} 0840+424$ respectively), similar to what one can expect in a cloud, while the farthest component is likely moving through an intercloud medium $\left(n_{\mathrm{ext}} \sim 0.02\right.$ and $0.08 \mathrm{~cm}^{-3}$, for $\mathrm{B} 0147+400$ and $\mathrm{B} 0840+424$ respectively) where the external density is about 3 orders of magnitude smaller.

Such clouds, indicating the presence of a rich and clumpy interstellar medium interacting with a CSS/GPS radio source, have been found by means of high-resolution spectral studies of the neutral hydrogen (Morganti et al. 2004; Labiano et al. 2006).

In the GPS ULIRG galaxy 4C 12.50, Morganti et al. (2004) detected a cloud with an H I mass of a few $10^{5}$ to $10^{6} M_{\odot}$ and $\sim 20 \times 66 \mathrm{pc}$ in size, corresponding to a density of $\sim 2 \times 10^{3} \mathrm{~cm}^{-3}$.

Labiano et al. (2006) studied the H I absorption in the two very asymmetric CSS sources 3C 49 and 3C 268.3, in which the brightest lobe is also the closest to the core, as in our targets.

In both sources, H I absorption was detected in the brightest (and closest) lobe only. The absorber medium has been interpreted in terms of clouds which are in the environment of the GPS/CSS radio source, with densities of $220 / c_{\mathrm{f}} \mathrm{cm}^{-3}$ $\left(0.04<c_{\mathrm{f}}<1\right.$, for $\left.3 \mathrm{C} 49\right)$ and $360 / c_{\mathrm{f}} \mathrm{cm}^{-3}\left(0.025<c_{\mathrm{f}}<1\right.$, for $3 \mathrm{C} 268.3)$, where $c_{\mathrm{f}}$ is the covering factor.

These values are in good agreement with the characteristics of the ISM in the Narrow Line Region (NLR; Fanti et al. 1995), in which the hot-spots of the aforementioned sources actually reside.

Our results on $\mathrm{B} 0147+400$ and $\mathrm{B} 0840+424$ are consistent with a picture in which one side of the radio source is strongly interacting with a dense cloud, while the other is expanding through an intercloud medium. The interaction with the cloud causes the lobe to propagate more slowly, and favours radio emission by means of compression and shocks (Jeyakumar et al. 2005; Bicknell et al. 2003), which increase the energy production efficiency. Furthermore, the clouds can also act as a Faraday screen, leading to the observed depolarization as found in both sources.

The detection of such asymmetric CSOs may be favoured by a selection effect. The interaction with a dense ambient medium may enhance the radio emission, making these objects more detectable. This is in agreement with other studies based on the asymmetries of CSOs (Saikia et al. 2003), in which on such a small scale, the probability that the brightest component is also the closest one to the core is higher than in larger sources.

\section{Conclusion}

We have presented the results of a new spectral analysis based on multi-frequency VLBA observations for two Compact 
Symmetric Objects from the B3-VLA-CSS sample (Fanti et al. 2001). The radiative ages derived from the analysis of the break frequency are of about $5 \times 10^{3}$ and $5 \times 10^{4}$ years, supporting the hypothesis that these are young objects. The individual hot-spot advance velocities range from $0.03 c$ to $0.3 c$ for the farthest lobe, and from 0.005 to 0.05 for the closest one.

The strong asymmetries in the arm ratio and brightness found in both sources are more likely due to a strong influence exerted by a clumpy and inhomogeneous medium, rather than a change in the hot-spot internal pressure. The brightest and closest component is partially and temporarily confined by a dense cloud which slows its propagation, while the other component is expanding through a more diluted ambient medium.

From this result, we infer that the knowledge of the properties of the ambient medium surrounding the radio source is of fundamental importance in order to draw a complete and reliable picture of the individual source evolution.

Acknowledgements. We like to thank the anonymous referee for carefully reading the manuscript and valuable suggestions. The VLBA is operated by the US National Radio Astronomy Observatory which is a facility of the National Science Foundation operated under a cooperative agreement by Associated Universities, Inc. This work has made use of the NASA/IPAC Extragalactic Database NED which is operated by the JPL, California Institute of Technology, under contract with the National Aeronautics and Space Administration.

\section{References}

Baum, S. A., O’Dea, C. P., de Bruyn, A. G., \& Murphy, D. W. 1990, A\&A, 232, 19

Bicknell, G. V., Saxton, C. J., \& Sutherland, R. S. 2003, PASA, 20, 102

Bolzonella, M., Miralles, J.-M., \& Pelló, R. 2000, A\&A, 363, 476

Dallacasa, D., Tinti, S., Fanti, C., et al. 2002, A\&A, 389, 115

Fanti, C., Fanti, R., Dallacasa, D., et al. 1995, A\&A, 302, 317

Fanti, C., Pozzi, F., Fanti, R., et al. 2000, A\&A, 358, 499

Fanti, C., Pozzi, F., Dallacasa, D., et al. 2001, A\&A, 369, 380

Fanti, C., Branchesi, M., Cotton, W. D., et al. 2004, A\&A, 427, 465

Gugliucci, N. E., Taylor, G. B., Peck, A. B., \& Giroletti, M. 2005, ApJ, 622, 136

Jaffe, W. J., \& Perola, G. C. 1974, A\&A, 26, 463

Jeyakumar, S., Wiita, P. J., Saikia, D. J., \& Hooda, J. S. 2005, A\&A, 432, 823

Kardashev, N. S. 1962, SvA, 6, 317

Labiano, A., Vermeulen, R. C., Barthel, C. P., et al. 2006, A\&A, 447, 481

Morganti, R., Oosterloo, T. A., Tadhunter, C. N., et al. 2004, A\&A, 424, 119

Murgia, M., Fanti, C., Fanti, R., et al. 1999, A\&A, 345, 769

Murgia, M. 2000, Ph.D. Thesis

Murgia, M. 2003, PASA, 20, 19

Norman, M. 1996, ASPC, 100, 405

Orienti, M., Dallacasa, D., Fanti, C., et al. 2004, A\&A, 426, 463

Pacholkczyk, A. G. 1970, Radio Astrophysics (San Francisco: Freeman \& Co.)

Polatidis, A. G., \& Conway, J. E. 2003, PASA, 20, 69

Readhead, A. C. S., Taylor, G. B., Xu, W., et al. 1996, ApJ, 460, 612

Saikia, D. J., Jeyakumar, S., Mantovani, F., et al. 2003, PASA, 20, 50

Siemiginowska, A., Cheung, C. C., LaMassa, S., et al. 2005, ApJ, 632, 110

Snellen, I. A. G., Schilizzi, R. T., Miley, G. K., et al. 2000, MNRAS, 319, 445

Trinchieri, G., Fabbiano, G., \& Canizares, C. R. 1986, ApJ, 310, 637

van Breugel, W., Miley, G., \& Heckman, T. 1984, AJ, 89, 5

Wilkinson, P. N., Polatidis, A. G., Readhead, A. C. S., et al. 1994, ApJ, 432, 87 